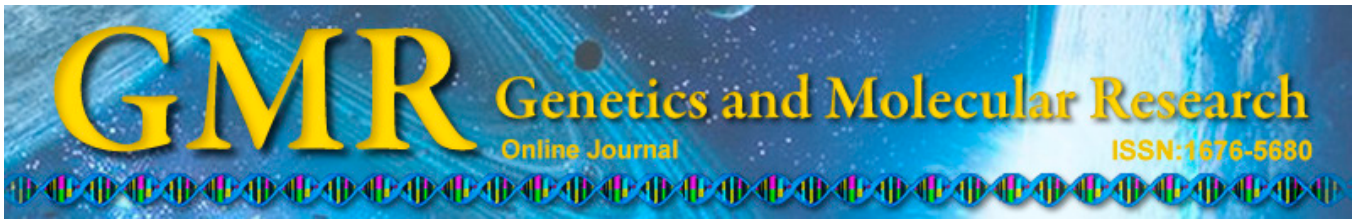

\title{
Propofol suppresses proliferation and invasion of pancreatic cancer cells by upregulating microRNA-133a expression
}

\author{
Z.T. Wang ${ }^{1}$, H.Y. Gong ${ }^{2}$, F. Zheng ${ }^{3}$, D.J. Liu ${ }^{1}$ and T.L. Dong ${ }^{1}$ \\ ${ }^{1}$ Department of Anesthesiology, \\ The Second Affiliated Hospital of Zhengzhou University, \\ Jinshui District, Zhengzhou, China \\ ${ }^{2}$ Department of Anesthesiology, \\ The First Affiliated Hospital of Xinxiang Medical University, Weihui, Henan, China \\ ${ }^{3}$ Department of Medical Ultrasonics, \\ The First Affiliated Hospital of Xinxiang Medical University Weihui, Henan, China \\ Corresponding author: T.L. Dong \\ E-mail: manudongt1@163.com
}

Genet. Mol. Res. 14 (3): 7529-7537 (2015)

Received November 4, 2014

Accepted March 26, 2015

Published July 3, 2015

DOI http://dx.doi.org/10.4238/2015.July.3.28

ABSTRACT. Propofol is a commonly used intravenous anesthetic. We evaluated its effects on the behavior of human pancreatic cancer cells and the underlying molecular mechanisms. The effects of propofol on Panc-1 cell proliferation, apoptosis, and invasion were determined by 3-(4,5-dimethylthiazol-2-yl)-2,5-diphenyltetrazolium bromide (MTT) assay, caspase-3 activity measurement, and Matrigel invasion assay. Quantitative polymerase chain reaction (qPCR) was used to assess microRNA-133a (miR-133a) expression. Anti-miR-133a was transfected into Panc-1 cells to assess the role of miR-133a in propofolinduced antitumor activity. Propofol significantly inhibited Panc-1 cell proliferation and invasion, and promoted apoptosis. Propofol also efficiently elevated miR-133a expression. Moreover, transfection of anti-miR-133a reversed the effects of propofol on the biological 
behavior of Panc-1 cells. Propofol can effectively inhibit proliferation and invasion, and induce apoptosis of pancreatic cancer cells, at least partly through the upregulation of miR-133a expression.

Key words: Propofol; Pancreatic cancer; miR-133a; Proliferation; Invasion

\section{INTRODUCTION}

Pancreatic cancer is the sixth leading cause of cancer-related death in China and has the lowest patient survival rate of any solid cancer (Guo and Cui, 2005). Despite advances in clinical and experimental oncology, the overall 5-year survival of patients with pancreatic cancer has increased only slightly from 3 to $5 \%$ (Jemal et al., 2010). The main reasons for suchprognoses include early metastatic spread, high local recurrence rate, and multifactorial resistance to treatments. Like other cancers, the development of pancreatic cancer is a multistep process with accumulation of genetic and epigenetic changes. Recent studies have revealed many pancreatic cancer-associated deregulated genes and signaling pathways (Chang et al., 2014; Ouaïssi et al., 2014), but the molecular mechanisms underlying the carcinogenesis, progression, and aggressiveness of the cancer have not been fully elucidated.

MicroRNAs (miRs) are a class of short (about 22 nucleotides in length), endogenous, single-stranded, non-protein-coding RNAs that directly bind to the 3'-untranslated regions (3'UTRs) of target messenger RNAs (mRNAs), leading to mRNA degradation or translational suppression (Bartel, 2009). It is now clear that miRs are involved in many different biological processes such as cell growth, apoptosis, development, differentiation, and endocrine homeostasis (Bartel, 2004). Accumulating research also suggests that miRs play an essential role in the biology of human cancers, and may provide a new and promising way to deal with cancer (Heneghan et al., 2010). Aberrant expression of miRs or mutations of miR genes have been well described in human pancreatic cancer (Bera et al., 2014; Shi et al., 2014; Song et al., 2014; Xu et al., 2014). It has been confirmed that downregulation of miR-133a may suppress tumors and many other types of human malignancy (Nohata et al., 2012). Qin et al. (2013) have reported that downregulation of miR-133a in pancreatic cancer correlates with large tumor size, advanced TNM stage, lymph node metastasis, vessel invasion, and shorter overall survival. Functional analyses showed that overexpression of miR-133a was able to reduce cell proliferation, invasion, and migration, promote cell apoptosis in vitro, and suppress tumorigenicity in vivo.

Propofol (2,6-diisopropylphenol, Figure 1) is a commonly used intravenous anesthetic. Apart from its multiple anesthetic advantages, propofol exerts a number of non-anesthetic effects (Vasileiou et al., 2009). There is increasing evidence to suggest a correlation between propofol and cancer inhibition. Propofol has the ability to inhibit the adhesion, proliferation, and invasion of cancer cells, and induce their apoptosis (Mammoto et al., 2002; Miao et al., 2010; Altenburg et al., 2011; Zhang et al., 2013b). Therefore, propofol might be a better agent than other anesthetics for cancer surgery (Inada et al., 2011). However, there is no available information on the antitumor action of propofol in pancreatic cancer cells. We aimed to investigate the effects of propofol on the biological behavior of human pancreatic cancer cells, and the related molecular mechanisms. 


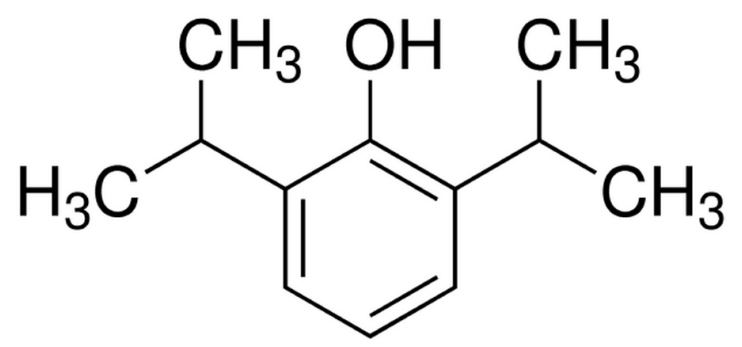

Figure 1. Chemical structure of propofol.

\section{MATERIAL AND METHODS}

\section{Cell culture and reagents}

Human pancreatic cancer cell line, Panc-1, was obtained from the Shanghai Institute of Cell Biology, the Chinese Academy of Sciences. Panc-1 was cultured in Dulbecco's modified Eagle's medium (DMEM) (Invitrogen, Carlsbad, California, USA), supplemented with $10 \%$ fetal bovine serum (St. Louis, MO, USA), $2 \mathrm{mM}$ glutamine, $100 \mathrm{U} / \mathrm{mL}$ penicillin, and $100 \mathrm{mg} / \mathrm{mL}$ streptomycin at $37^{\circ} \mathrm{C}$ in a $5 \% \mathrm{CO}_{2}$ atmosphere. Propofol was acquired from Sigma Aldrich Chemical Co. (Sigma, St. Louis, MO, USA) and diluted with dimethyl sulfoxide (Sigma) for in vitro assays.

\section{Cell viability assay}

Cell viability was determined using a3-(4,5-dimethylthiazol-2-yl)-2,5-diphenyltetrazolium bromide (MTT) (Sigma) assay. Briefly, the cells were seeded at a density of $5 \times 10^{3}$ cells/well on 96-well plates containing $180 \mu \mathrm{L}$ culture medium and incubated at $37^{\circ} \mathrm{Cin} 5 \%$ $\mathrm{CO}_{2}$ overnight. At the indicated time after treatment, $20 \mu \mathrm{L}$ MTT $(5 \mathrm{mg} / \mathrm{mL})$ was added into each corresponding test well and incubated for $4 \mathrm{~h}$. The reaction was then solubilized by adding $200 \mu \mathrm{L}$ dimethyl sulfoxide to each well. Optical density (OD) was evaluated on a multidetection microplate reader (BMG LABTECH, Durham, NC, USA) by measuring the absorbance at a wavelength of $570 \mathrm{~nm}$. The experiments were repeated three times independently and the results are reported as means \pm standard deviation (SD).

\section{Apoptosis analysis by Hoechst 33258 staining and caspase-3 activity measurement}

First, Hoechst 33258 staining was used to identify apoptotic Panc-1 cells. Briefly, cells were grown on cover slides on 6-well plates. After drug treatment, cells were fixed with 4\% paraformaldehyde and stained with Hoechst 33258 (Sigma) for 5 min. Nuclear morphology was observed under a fluorescence microscope (IX51, Olympus). Dead cells and apoptotic bodies were identified by condensed or fragmented nuclei.

To detect cell apoptosis at the molecular level, caspase-3 activity was also measured using a caspase colorimetric protease assay. Briefly, cells were cultured on 96-well plates, treated with drugs, and analyzed using a Caspase-Glo 3/7 Assay kit (Promega, USA) following the manufacturer instructions. 


\section{Matrigel invasion assay}

Invasion assays were performed in triplicate using a 24-well invasion chamber system coated with Matrigel (50 $\mu \mathrm{L}$ per-filter) (BD Biosciences, Bedford, MA, USA). Cells were seeded in the upper chamber at a concentration of $1 \times 10^{5}$ cells/well in serum-free DMEM. DMEM containing $10 \%$ fetal bovine serum was added to the lower chambers as a chemoattractant. After incubation for $24 \mathrm{~h}$, non-migratory cells in the upper chamber were removed using a cotton-tip applicator. Migrated cells on the lower surface were fixed with $95 \%$ ethanol, stained with $0.1 \%$ crystal violet, and counted under a microscope (Olympus Corp., Tokyo, Japan).

\section{Detection of miRNA expression by quantitative polymerase chain reaction (PCR)}

After treatment without (control group) or with propofol for $24 \mathrm{~h}$, approximately $5 \mathrm{x}$ $10^{6}$ cells were collected and miRNAs were extracted using TRIzol reagent according to the manufacturer instructions (Invitrogen, USA). The miR-133a level was determined by quantitative PCR using TransStart ${ }^{\mathrm{TM}}$ SYBR Green qPCR Supermix (TransGen Biotech, Beijing, China) and U6 small nuclear RNA was used as an endogenous reference gene for normalization. For miR-133a, the primers were as follows: forward, 5'-CTGCATTGGTCCCCTTCAAC-3' and reverse, 5'-CAGTGCAGGGTCCGAGGTAT-3'. For U6, the primers were as follows: forward 5'-CTCGCTTCGGCAGCACA-3' and reverse, 5'-AACGCTTCACGAATTTGCGT-3'. The threshold cycle $(\mathrm{Ct})$ was defined as the fractional cycle number at which the fluorescence passed the fixed threshold. Each sample was measured in triplicate, and the relative amount of miR-133a to U6 was calculated using the equation $2^{-\Delta \mathrm{Ct}}$, where $\Delta \mathrm{Ct}=\left(\mathrm{Ct}^{\mathrm{miR}-133 \mathrm{a}}-\mathrm{Ct}^{\mathrm{U} 6}\right)$.

\section{Cell transfection}

To selectively downregulate miR-133a, Panc-1 cells were seeded into each well of a 24-well plate and incubated overnight, then transfected with anti-miR-133a (GenePharma, Shanghai, China) at a concentration of $50 \mathrm{nM}$ using Lipofectamine 2000 (Invitrogen, CA, USA). The cells were harvested for further analysis $24 \mathrm{~h}$ after transfection.

\section{Statistics}

Data are reported as means $\pm \mathrm{SD}$. Statistical analysis was performed using the SPSS version 17.0 software (SPSS Inc., Chicago, IL, USA). Differences between groups were assessed by unpaired Student $t$-test. All tests were two-tailed, and the significance level was set at $\mathrm{P}<0.05$.

\section{RESULTS}

\section{Effects of propofol on cell proliferation, apoptosis, and invasion}

First, we investigated the effects of propofol on cell proliferation, apoptosis, and invasion. The Panc-1 cells were cultured in different concentrations of propofol and the cell proliferation was examined using MTT analysis. As shown in Figure 2A, the proliferation of Panc-1 was inhibited by propofol in dose- and time-dependent ways. Propofol at concentrations of 5 and $10 \mu \mathrm{g} / \mathrm{mL}$ notably inhibited the proliferation at 48 and $72 \mathrm{~h}$. To further detect 
cell apoptosis, Hoechst 33258 staining and caspase-3 activity were investigated in our study. After exposure to propofol for $48 \mathrm{~h}$, Panc-1 cells exhibited increasing apoptosis, as indicated in Figure 2B and $\mathrm{C}$. The Matrigel invasion assay also revealed that propofol significantly reduced cell invasion at concentrations of 5 and $10 \mu \mathrm{g} / \mathrm{mL}$ (Figure 2D). Collectively, propofol inhibits proliferation and invasion, and promotes apoptosis of Panc-1 cells.
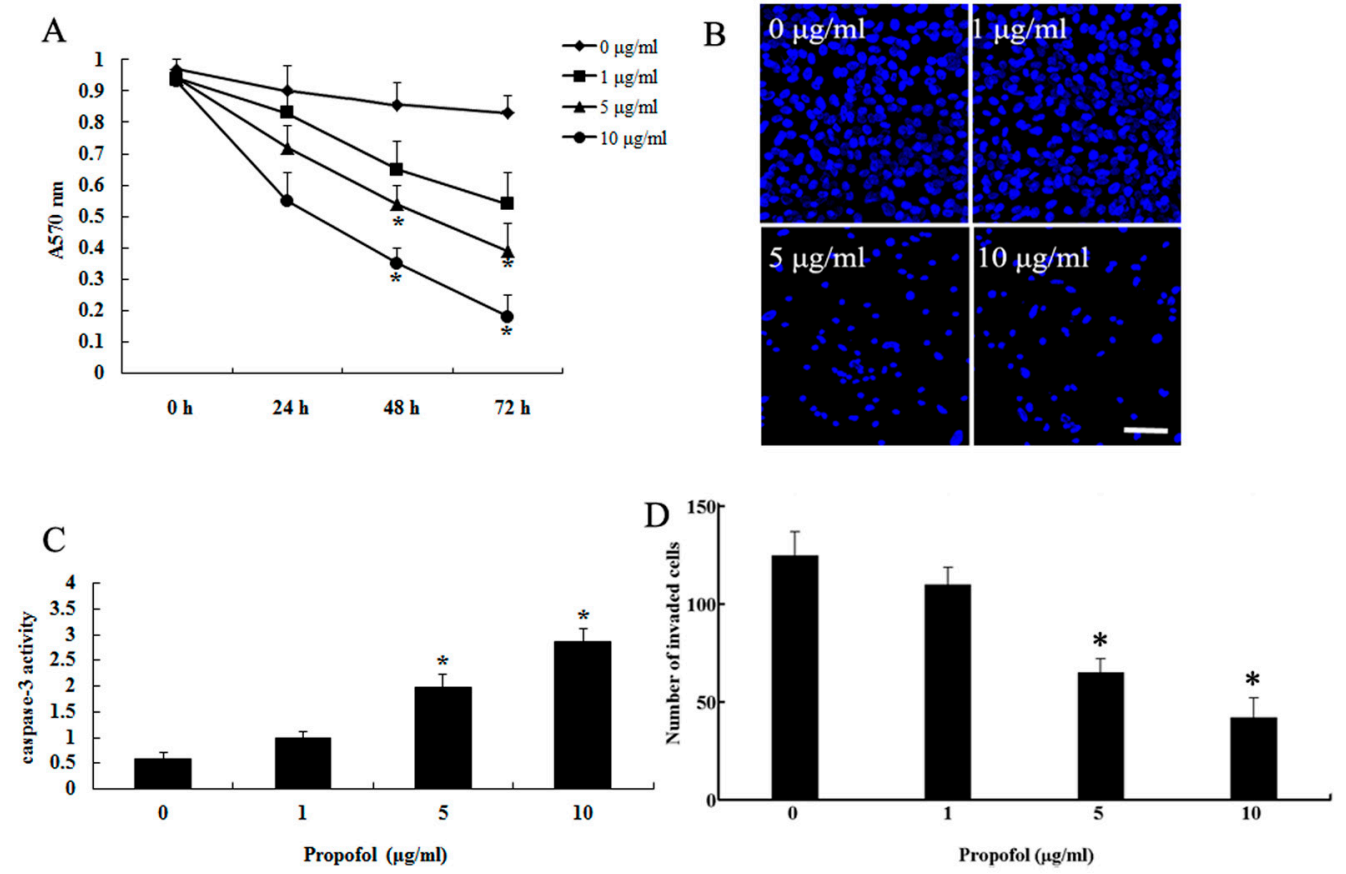

Figure 2. Propofol inhibits cell proliferation, promotes apoptosis, and reduces invasion. Propofol treatment inhibited proliferation (A), promoted apoptosis (B and $\mathbf{C})$, and reduced invasion (D) of the pancreatic cancer cell line Panc-1 in a dose-dependent manner. ${ }^{*} \mathrm{P}<0.01$ compared with the control group without propofol treatment.

\section{Propofol stimulates miR-133a expression}

As shown in Figure 3, propofol treatment enhanced miR-133a expression in Panc-1 cells in a dose-dependent manner. More specifically, propofol at concentrations of 5 and 10 $\mu \mathrm{g} / \mathrm{mL}$ elevated miR-133a expression in Panc- 1 cells by 2.46 - and 3.95 -fold, respectively.

\section{miR-133a elimination reverses the effects of propofol on cell proliferation, apoptosis, and invasion}

To further explore the role of miR-133a in the effect of propofol in Panc-1 cells, antimiR-133a was used to eliminate miR-133a. First, anti-miR-133a notably reduced the expression of miR-133a, suggesting that anti-miR-133a successfully penetrated into Panc-1 cells (Figure 4A). Moreover, the inhibitory effects of propofol $(10 \mu \mathrm{g} / \mathrm{mL})$ on cell proliferation and 
invasion, and its promotion of apoptosis, were significantly reversed after transfection with anti-miR-133a (Figure 4B-E).

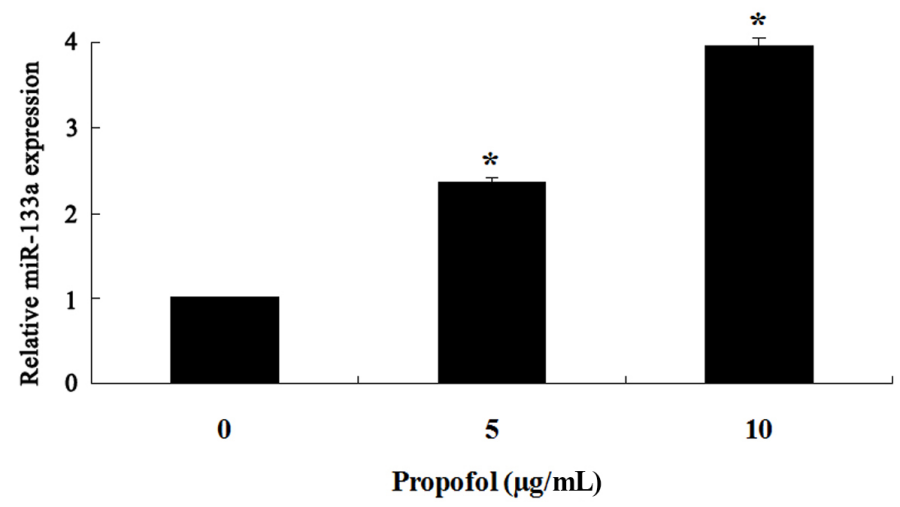

Figure 3. Propofol stimulates microRNA-133a (miR-133a) expression. Propofol treatment increased the expression of miR-133a in a dose-dependent manner. ${ }^{*} \mathrm{P}<0.01$ compared with the control group without propofol treatment.

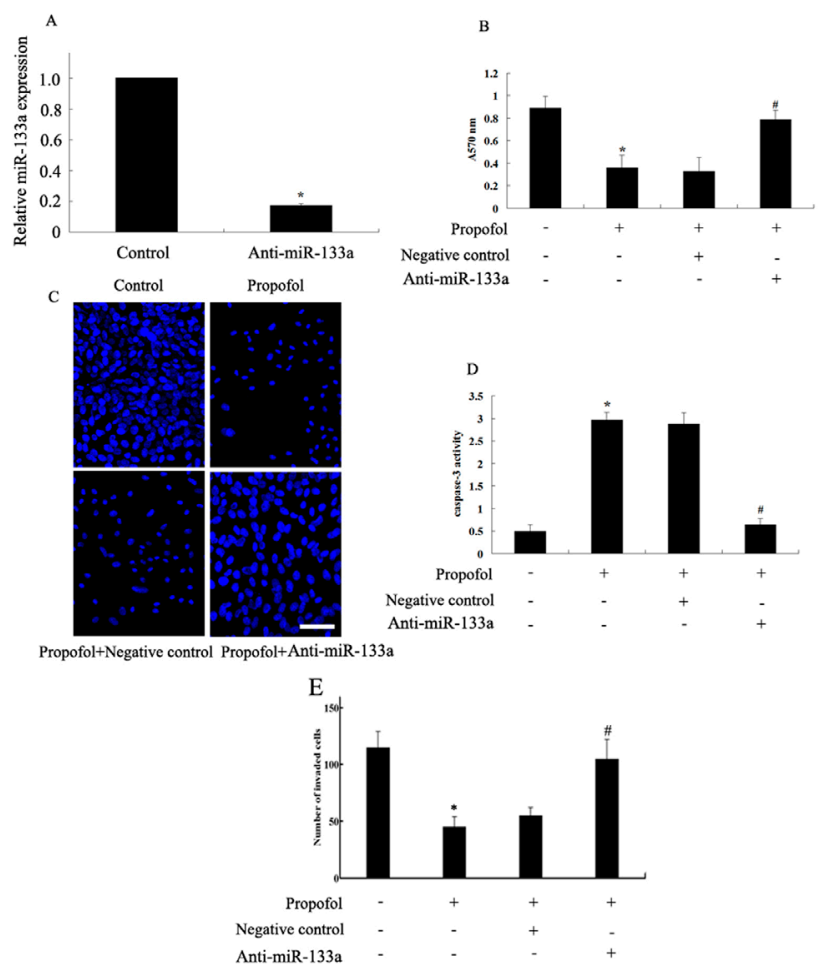

Figure 4. Anti-microRNA-133a (anti-miR-133a) can reverse the effect of propofol. Anti-miR-133a significantly reduced the expression of miR-133a in Panc-1 cells (A). Anti-miR-133a evidently promoted cell proliferation (B), inhibited cell apoptosis $(\mathbf{C}$ and $\mathbf{D})$, and enhanced invasion $(\mathbf{E})$ after treatment with propofol. ${ }^{*} \mathrm{P}<0.01$ compared with the control group without propofol treatment and ${ }^{\#} \mathrm{P}<0.01$ compared with the propofol-treated group transfected with the negative control. 


\section{DISCUSSION}

Pancreatic cancer is one of the most lethal of all human cancers. Although the cancer death rates of most malignancies have decreased owing to improvements in early detection and treatment, the clinical outcome of patients with pancreatic cancer is still poor. In this study, we evaluated the effects of propofol on the behavior of human pancreatic cancer cells and found that propofol inhibited Panc-1 cell proliferation and invasion, and promoted apoptosis. Our results were consistent with those of other studies. For example, Mammoto et al. (2002) have demonstrated that clinically relevant concentrations of propofol reduce the invasion capability of human cancer cells (HeLa, HT1080, HOS, and RPMI-7951). Miao et al. (2010) have reported that propofol inhibits the invasion of LOVO colon cancer cells. Zhang et al. (2013a,b) have shown that propofol can effectively induce apoptosis and reduce the invasiveness of hepatocellular carcinoma cells. Taken together, this evidence suggests that propofol may be a particularly suitable anesthetic for the peri-operative phase in cancer surgery (Siddiqui et al., 2005; Inada et al., 2011; Wu et al., 2013).

The discovery of miRs has substantially changed the view of gene regulation, and new findings over the past few years have catapulted miRNAs to the center stage of cancer molecular biology. To clarify the mechanism involved in the suppression of Panc-1 cells, the effect of propofol on miR-133a expression was examined. We observed that propofol stimulated the expression of miR-133a in Panc-1 cells. More importantly, neutralizing miR-133a with antimiR-133a transfection reversed the effect of propofol on the proliferation, apoptosis, and invasion of Panc-1 cells. These results suggest that the antitumor effect of propofol on pancreatic cancer cells may be partly due to the upregulation of miR-133a. miR-133a downregulation has been confirmed in lung cancer (Moriya et al., 2012), breast cancer (Wu et al., 2012), rhabdomyosarcoma (Missiaglia et al., 2010), maxillary sinus squamous cell carcinoma (Nohata et al., 2011), tongue cancer (Wong et al., 2008), esophageal cancer (Kano et al., 2010), gastric cancer (Qiu et al., 2014), prostate cancer (Kojima et al., 2012), ovarian cancer (Guo et al., 2014), renal cell carcinoma (Kawakami et al., 2012), and pancreatic cancer (Qin et al., 2013). In these cancers, decreased miR-133a expression results in the induction of cell proliferation, the inhibition of apoptosis, or the promotion of cell invasion, indicating that miR-133a acts as a tumor-suppressor miRNA. Previous studies have shown that propofol may affect the biological behaviors of cancer cells by regulation of miRNA expression. Zhang et al. (2013a,b) found that propofol can effectively induce apoptosis and inhibit the adhesion of hepatocellular carcinoma cells by upregulating miR-199a. A recent study reported that propofol inhibits proliferation and invasion of osteosarcoma cells through miR-143 upregulation (Ye et al., 2014). However, the detailed mechanisms by which propofol influences miRNA expression are still unclear, and further clarification is needed.

In conclusion, our study provides new insights into the effect of propofol on the behavior of pancreatic cancer cells and the mechanisms involved. The results suggest that propofol can inhibit proliferation and invasion, and induce apoptosis in Panc-1 cells, and modulation of miR-133a may contribute to this antitumor action. Further studies are needed to validate the clinical relevance of these findings.

\section{Conflicts of interest}

The authors declare no conflict of interest. 


\section{REFERENCES}

Altenburg JD, Harvey KA, McCray S, Xu Z, et al. (2011). A novel 2,6-diisopropylphenyl-docosahexaenoamide conjugate induces apoptosis in T cell acute lymphoblastic leukemia cell lines. Biochem. Biophys. Res. Commun. 411:427-432.

Bartel DP (2004). MicroRNAs: genomics, biogenesis, mechanism, and function. Cell 116: 281-297.

Bartel DP (2009). MicroRNAs: target recognition and regulatory functions. Cell 136: 215-233.

Bera A, VenkataSubbaRao K, Manoharan MS, Hill P, et al. (2014). A miRNA signature of chemoresistant mesenchymal phenotype identifies novel molecular targets associated with advanced pancreatic cancer. PLoS One 9: e106343.

Chang ZY, Sun R, Ma YS, Fu D, et al. (2014). Differential gene expression of the key signalling pathway in paracarcinoma, carcinoma and relapse human pancreatic cancer. Cell Biochem. Funct. 32: 258-267.

Guo X and Cui Z (2005). Current diagnosis and treatment of pancreatic cancer in China. Pancreas 31: 13-22.

Guo J, Xia B, Meng F and Lou G (2014). miR-133a suppresses ovarian cancer cell proliferation by directly targeting insulin-like growth factor 1 receptor. Tumour Biol. 35: 1557-1564.

Heneghan HM, Miller N and Kerin MJ (2010). MiRNAs as biomarkers and therapeutic targets in cancer. Curr. Opin. Pharmacol. 10: 543-550.

Inada T, Kubo K and Shingu K (2011). Possible link between cyclooxygenase-inhibiting and antitumor properties of propofol. J. Anesth. 25: 569-575.

Jemal A, Siegel R, Xu J and Ward E (2010). Cancer statistics, 2010. CA Cancer J. Clin. 60: 277-300.

Kano M, Seki N, Kikkawa N, Fujimura L, et al. (2010). miR-145, miR-133a and miR-133b: Tumor-suppressive miRNAs target FSCN1 in esophageal squamous cell carcinoma. International journal of cancer. Int. J. Cancer 127: 2804-2814.

Kawakami K, Enokida H, Chiyomaru T, Tatarano S, et al. (2012). The functional significance of miR-1 and miR-133a in renal cell carcinoma. Eur. J. Cancer 48: 827-836.

Kojima S, Chiyomaru T, Kawakami K, Yoshino H, et al. (2012). Tumour suppressors miR-1 and miR-133a target the oncogenic function of purine nucleoside phosphorylase (PNP) in prostate cancer. Br. J. Cancer 106: 405-413.

Mammoto T, Mukai M, Mammoto A, Yamanaka Y, et al. (2002). Intravenous anesthetic, propofol inhibits invasion of cancer cells. Cancer Lett. 184: 165-170.

Miao Y, Zhang Y, Wan H, Chen L, et al. (2010). GABA-receptor agonist, propofol inhibits invasion of colon carcinoma cells. Biomed. Pharmacother. 64: 583-588.

Missiaglia E, Shepherd CJ, Patel S, Thway K, et al. (2010). MicroRNA-206 expression levels correlate with clinical behaviour of rhabdomyosarcomas. Br. J. Cancer 102: 1769-1777.

Moriya Y, Nohata N, Kinoshita T, Mutallip M, et al. (2012). Tumor suppressive microRNA-133a regulates novel molecular networks in lung squamous cell carcinoma. J. Hum. Genet. 57: 38-45.

Nohata N, Hanazawa T, Kikkawa N, Sakurai D, et al. (2011). Identification of novel molecular targets regulated by tumor suppressive miR-1/miR-133a in maxillary sinus squamous cell carcinoma. Int. J. Oncol. 39: 1099-1107.

Nohata N, Hanazawa T, Enokida H and Seki N (2012). microRNA-1/133a and microRNA-206/133b clusters: dysregulation and functional roles in human cancers. Oncotarget 3: 9-21.

Ouaïssi M, Silvy F, Loncle C, Ferraz da Silva D, et al. (2014). Further characterization of HDAC and SIRT gene expression patterns in pancreatic cancer and their relation to disease outcome. PLoS One 9: e108520.

Qin Y, Dang X, Li W and Ma Q (2013). miR-133a functions as a tumor suppressor and directly targets FSCN1 in pancreatic cancer. Oncol. Res. 21: 353-363.

Qiu T, Zhou X, Wang J, Du Y, et al. (2014). MiR-145, miR-133a and miR-133b inhibit proliferation, migration, invasion and cell cycle progression via targeting transcription factor Sp1 in gastric cancer. FEBS Lett. 588: 1168-1177.

Shi S, Lu Y, Qin Y, Li W, et al. (2014). miR-1247 is correlated with prognosis of pancreatic cancer and inhibits cell proliferation by targeting neuropilins. Curr. Mol. Med. 14: 316-327.

Siddiqui RA, Zerouga M, Wu M, Castillo A, et al. (2005). Anticancer properties of propofol-docosahexaenoate and propofol-eicosapentaenoate on breast cancer cells. Breast Cancer Res. 7: R645-R654.

Song Z, Ren H, Gao S, Zhao X, et al. (2014). The clinical significance and regulation mechanism of hypoxia-inducible factor-1 and miR-191 expression in pancreatic cancer. Tumour Biol. 35: 11319-11328.

Vasileiou I, Xanthos T, Koudouna E, Perrea D, et al. (2009). Propofol: a review of its non-anaesthetic effects. Eur. J. Pharmacol. 605: 1-8.

Wong TS, Liu XB, Chung-Wai Ho A, Po-Wing Yuen A, et al. (2008). Identification of pyruvate kinase type M2 as potential oncoprotein in squamous cell carcinoma of tongue through microRNA profiling. Int. J. Cancer 123: 251257.

Wu ZS, Wang CQ, Xiang R, Liu X et al. (2012). Loss of miR-133a expression associated with poor survival of breast cancer and restoration of miR-133a expression inhibited breast cancer cell growth and invasion. BMC Cancer 12: 51.

Genetics and Molecular Research 14 (3): $7529-7537$ (2015)

CFUNPEC-RP www.funpecrp.com.br 
Wu RS, Liu KC, Tang NY, Chung HK, et al. (2013). cDNA microarray analysis of the gene expression of murine leukemia RAW 264.7 cells after exposure to propofol. Environ. Toxicol. 28: 471-478.

Xu J, Wang T, Cao Z, Huang H, et al. (2014). MiR-497 downregulation contributes to the malignancy of pancreatic cancer and associates with a poor prognosis. Oncotarget 5: 6983-6993.

Ye Z, Jingzhong L, Yangbo L, Lei C, et al. (2014). Propofol inhibits proliferation and invasion of osteosarcoma cells by regulation of microRNA-143 expression. Oncol. Res. 21: 201-207.

Zhang J, Wu GQ, Zhang Y, Feng ZY, et al. (2013a). Propofol induces apoptosis of hepatocellular carcinoma cells by upregulation of microRNA-199a expression. Cell Biol. Int. 37: 227-232.

Zhang J, Zhang D, Wu GQ, Feng ZY, et al. (2013b). Propofol inhibits the adhesion of hepatocellular carcinoma cells by upregulating microRNA-199a and downregulating MMP-9 expression. Hepatobiliary Pancreat. Dis. Int. 12: 305-309. 